\title{
L'intérieur institutionnel : entretien avec Lorna Rhodes
}

\author{
Entretien conduit par Camille Lancelevée et Livia Velpry \\ The institutional interior: interview with Lorna Rhodes
}

Lorna Rhodes, Camille Lancelevée et Livia Velpry

\section{NOTE DE L'AUTEUR}

Traduction réalisée par Charlotte Nordmann et financée par l'ANR Contrast

Dans un univers académique où règnent la course à la productivité, l'injonction à la cohérence des parcours et le formatage croissant des travaux, Lorna Rhodes occupe une place singulière. Sa carrière s'organise autour de trois ethnographies longues portant sur des sujets éloignés. Après un séjour dans un village au Sri Lanka, qui donne lieu à sa thèse sur les rituels de guérison, elle fréquente longuement, dans les années 1980, un service d'urgence psychiatrique situé à Baltimore (Maryland), puis pénètre dans les années 1990, comme anthropologue, dans le quartier de haute sécurité d'une prison de l'État de Washington. De ces longues immersions dans le monde de la psychiatrie et de la prison, Lorna Rhodes tire principalement deux ouvrages finement ciselés : Emptying Beds (Rhodes, 1991) et Total Confinement (Rhodes, 2004). Dans le premier, elle décrit le changement de paradigme alors à l'œuvre dans la psychiatrie publique américaine, où la normalisation biomédicale accompagne la transformation de l'hôpital en un lieu de passage, et non plus de vie. Dans le second livre, elle rend compte des mécanismes par lesquels la société américaine rationalise une mort sociale, celle des personnes placées dans les unités de sécurité maximum.

2 Malgré une apparente dispersion, ces travaux successifs laissent apparaître la cohérence d'une " œuvre » : Lorna Rhodes y construit une réflexion sur la façon dont les acteurs se démènent au sein de mondes sociaux clos où s'exerce un mandat de contrôle social, afin de résoudre les problèmes qui surgissent, de faire fonctionner les 
institutions en charge de ce contrôle ou tout simplement de s'y frayer un chemin. À la façon d'un peintre impressionniste, Lorna Rhodes rend compte, par petites touches éclairantes, du quotidien des institutions psychiatrique et carcérale, là où se manifestent avec force les tensions morales entre soin et contrôle, entre attention et surveillance, entre gestion des corps et gestion des populations. Ce faisant, elle mêle avec souplesse richesse empirique et discussion théorique. En donnant à voir l'énergie déployée, ici pour faire sortir un patient, là pour justifier la contrainte qui pèse sur un détenu, elle parvient à montrer comment l'institution psychiatrique se détourne des plus précaires, ou encore comment l'enfermement paroxystique du Supermax ${ }^{1}$ produit la violence qu'il entendait neutraliser. Sans jamais verser dans une dénonciation de principe ou réduire le réel à un paradigme englobant - «tournant punitif », "gestion des risques », «néolibéralisme» ou autre -, son œuvre offre pourtant des prises à la critique du pouvoir institutionnel grâce à l'attention constante portée aux ressorts de ce qu'elle décrit comme de "petites sociétés perverses ».

3 Si ces deux contributions importantes à l'anthropologie américaine n'ont jusqu'à présent pas été traduites en français, à l'exception d'un article (Rhodes, 2015), elles ont néanmoins influencé en France les travaux de sociologues de la psychiatrie comme de la prison. Lus et repris quelques années après leur parution, Emptying beds et Total Confinement sont venus à chaque fois nourrir des champs qui se trouvaient alors en plein «tournant ethnographique ", avec le développement d'enquêtes par observation au sein de l'hôpital psychiatrique et de la prison ${ }^{2}$. Il nous paraissait indispensable, dans un numéro sur les liens entre champ pénal et psychiatrie, de proposer un retour sur le parcours de cette anthropologue, dont l'œuvre traverse ces univers sociaux et propose un regard transversal sur les formes d'institutionnalisation ${ }^{3}$ du traitement des marges sociales.

Plus que de restituer le contenu de ses ouvrages, nous avons voulu revenir sur l'équilibre délicat qu'elle réussit à créer dans ses travaux, entre critique des institutions totales et compréhension des vies qui s'y déroulent. Comment, pour tenir cet équilibre, se confronter à la pensée des auteurs les plus critiques tout en maintenant entière la complexité des réalités observées ? Certes, la description rend possible la dénonciation, et, comme le souligne Lorna Rhodes, Michel Foucault lui-même laisse advenir la critique à partir d'une description du monde. Cependant, la confrontation aux situations empiriques est toujours déstabilisante: le réel produit un effet kaléidoscopique ${ }^{4}$. Lorna Rhodes l'aborde avec humilité, rappelant sa dette vis-à-vis des personnes rencontrées sur le terrain, mais aussi les limites intrinsèques aux efforts des sciences sociales pour objectiver la réalité. Le soin et la subtilité avec lesquels elle rend compte dans ses ouvrages des tensions morales qui imprègnent ces institutions lui permettent de rester sur une étroite ligne de crête, sans jamais verser ni dans la dénonciation idéologique ni dans la description tautologique. La posture épistémologique singulière de Lorna Rhodes s'incarne tout particulièrement dans son style d'écriture, qui imbrique de façon étroite de denses descriptions ethnographiques avec la mobilisation de notions et discussions théoriques. Nous avons donc aussi souhaité qu'elle raconte concrètement les processus qui aboutissent à créer une intense expérience de lecture, à la fois émotionnelle et intellectuelle. 


\section{Ma carrière n'a été qu'une succession d'accidents}

[Livia Velpry] - De Emptying Beds à Total Confinement, on connaît bien vos travaux sur la psychiatrie et la prison aux États-Unis. Mais votre première recherche a été menée au Sri Lanka. Commençons donc par le commencement : comment décririez-vous votre parcours de chercheuse?

[Lorna Rhodes] - J'ai fait mes études à l'université de Cornell et, dès la première année, j'ai pris la décision d'aller en Asie du Sud. Cette décision avait des motifs personnels : j'étais mariée à quelqu'un qui venait du Sri Lanka, et nous souhaitions tous deux y aller. Par ailleurs, j'étais intéressée par le bouddhisme et plus généralement par la religion, et c'était l'endroit idéal pour approfondir ces questions. Il y avait aussi des spécialistes de l'Asie du Sud à Cornell, et en particulier quelqu'un qui enseignait le cingalais, de sorte que je pouvais apprendre la langue. Au Sri Lanka, j'ai étudié les rituels de guérison. Il s'agissait de mises en scène organisées autour d'un patient qui était au centre de la performance, laquelle impliquait également les personnages démoniaques ayant causé la maladie. Je vivais dans les Hauts plateaux $\mathrm{du}$ Sri Lanka, au centre de l'île, où se pratiquait une version particulière de cette danse. J'habitais dans un village et j'assistais à ces rituels de guérison qui duraient toute la nuit, et c'est sur ce sujet qu'a porté ma thèse. À cette époque, en anthropologie, on considérait généralement qu'il fallait apprendre une autre langue et partir ailleurs. On ne nous donnait aucune formation pour le travail de terrain: l'idée était qu'une fois là-bas, on trouverait comment s'y prendre. D'un certain point de vue, c'était une expérience de terrain classique - je vivais seule dans un village, mais ma relation au pays était différente parce que je l'avais pour ainsi dire épousé.

Lorsque je suis revenue aux États-Unis, j'ai eu quelques brefs contrats d'enseignement. Je travaillais toujours sur l'Asie du Sud sous l'angle des «mythes, rituels et symboles ». J'étais très influencée par le travail de Clifford Geertz mais aussi par celui de Victor Turner, deux figures influentes à l'époque. Je ne connaissais pas l'anthropologie médicale, qui d'ailleurs n'existait alors pour ainsi dire pas. Lorsque l'on consulte les résumés des communications aux conférences de l'Association américaine d'anthropologie de la fin des années 1960, on constate que les questions étaient beaucoup plus larges, qu'elles couvraient un territoire à la fois plus vaste et moins défini... C'était aussi ma manière de penser à l'époque. Bien sûr, à y réfléchir maintenant, il y a énormément de choses que je n'ai pas vues, comme la situation postcoloniale dans laquelle je travaillais : je n'avais tout simplement pas les outils pour la voir.

Et puis, au début des années 1970, j'ai obtenu un post-doc dans un endroit minuscule qui faisait partie de la Harvard Medical School, la faculté de médecine de Harvard, et était soutenu par le National Institute of Mental Health : le Laboratoire de psychiatrie sociale. Lorsque j'ai commencé à travailler là-bas, je pensais faire quelque chose en rapport avec les immigrés indiens présents à Boston. Je voulais savoir quelles pratiques médicales les femmes avaient apportées avec elles, si elles avaient encore des pratiques rituelles importantes. Mais arrivée à Harvard, j'ai découvert que le laboratoire se trouvait en face du Centre de santé mentale du Massachusetts. C'est un vieil hôpital, relativement connu aux États-Unis, et qui avait jusque-là surtout travaillé avec la psychanalyse. Au moment où je suis arrivée, ils étaient en train de basculer vers le paradigme biologique en psychiatrie ${ }^{5}$. Comme j'étais en post-doc, je 
pouvais m'y rendre et y passer du temps un peu comme je voulais. C'est ce qui a éveillé mon intérêt pour la psychiatrie. J'ai commencé à assister aux réunions scientifiques, je me suis fait des amis parmi les résidents... Je pouvais observer la vie de l'hôpital de l'intérieur.

[Camille Lancelevée] - C'est donc dans les années 1970 que vous vous tournez vers l'anthropologie médicale. Comment se passe ce changement d'objet et de regard?

[LR] - Jusqu'à ce post-doc, mon parcours a été classique. Mais après, pendant les années 1970, je n'ai plus occupé de poste de recherche à proprement parler. Au départ, j'étais à Boston, et ensuite j'ai commencé à enseigner à des étudiants en médecine au département de psychiatrie de l'université du Maryland, à Baltimore. Il se trouvait juste à côté de l'hôpital psychiatrique où j'ai mené la recherche qui a abouti à la publication de Emptying Beds, en 1991. Le directeur de l'hôpital m'a recrutée en me disant tout simplement : «Essayez de voir ce que vous pouvez avoir à dire de notre service d'urgence. " J'ai donc passé environ deux ans à observer ce service, avec des interruptions. Parallèlement, je donnais un cours d'introduction aux sciences sociales aux étudiants de médecine de première année. Mon statut était précaire, mais le travail était intéressant. C'est vers cette période qu'il est apparu clairement que l'anthropologie médicale avait évolué, et que c'était ce que je faisais.

Si j'avais poursuivi dans la voie toute tracée qui s'offrait à moi, j'aurais continué à faire de l'anthropologie interprétative. Les premières années d'Université sont déterminantes pour notre socialisation. Mais dans mon cas, il y a eu cette dizaine d'années passée dans les hôpitaux, qui m'a amenée à chercher comment comprendre ce que je voyais. Tout au long des années 1980, il existait par ailleurs une tension entre l'anthropologie interprétative et des façons de penser plus post-modernes. Cela correspondait aussi à un renouvellement générationnel, ce qui arrive fréquemment en anthropologie.

Ensuite, au moment où l'anthropologie médicale était en train de devenir un champ à part entière, j'étais à l'intérieur du monde de l'hôpital. Ma carrière n'a été qu'une succession d'accidents... Je veux dire par là que des opportunités ont surgi là où je ne les attendais pas, et que j'ai été en mesure de creuser des sujets qui, à l'époque, ne paraissaient pas du tout constitués. Quand j'ai été recrutée par l'université de Washington, au début des années 1980, c'était parce qu'ils cherchaient quelqu'un en anthropologie médicale, et que mon travail allait dans ce sens. Arthur Kleinman avait fait partie de leur département de psychiatrie, et il avait tissé des liens forts avec le département d'anthropologie. Lorsqu'il est parti, l'université a cherché à recruter un anthropologue médical qui aurait une expérience en hôpital. C'est vraiment à ce moment-là que je suis devenue officiellement une « anthropologue médicale »!

C'est encore une opportunité qui m'a entraînée vers l'univers des Supermax. Quand je suis arrivée à l'université de Washington, je me suis concentrée sur l'enseignement et l'écriture de mon livre. Six ou sept ans plus tard, à peu près au moment où j'ai obtenu ma titularisation, un groupe de personnes a engagé un projet sur la prison et je me suis jointe à eux. Au départ, nous étions très nombreux, et puis, au bout de quelques années, nous n'étions plus que trois : un philosophe, un travailleur social et moi. On travaillait ensemble avec le système carcéral, avec les personnes qui le géraient et y travaillaient, sur divers problèmes, sur des questions très pratiques. Puis j'ai commencé à me rendre seule dans les prisons, pour y faire un travail d'ethnographie. Le projet s'est développé par lui-même, ce n'est pas un projet de recherche que 
j'aurais planifié à l'avance. Ma recherche portait sur les personnes souffrant de pathologie mentale en prison, de sorte que mon parcours a pris une cohérence qui n'était en rien planifiée.

\section{La façon dont les gens se rassemblent autour d'un problème}

[CL] - Alors comment décririez-vous cette cohérence ? Quel pourrait être, à vos yeux, le fil rouge, le thème fondamental qui court à travers vos différents terrains, du Sri Lanka aux prisons de très haute sécurité ?

[LR] - Votre question est intéressante... Vous savez, lorsque j'étais au Centre de santé mentale du Massachusetts, en train de parler aux résidents, j'ai pris conscience que la situation n'était pas si éloignée de celles que j'étudiais au Sri Lanka : dans les rituels de guérison sri-lankais, il y a une personne malade et, ce qui est passionnant, c'est la façon dont tout le monde se rassemble autour d'elle et construit un monde pour et avec elle, un monde structuré autour d'elle. Un hôpital psychiatrique, c'est la même chose, ou du moins ça l'était à l'époque. Le patient est au centre, et la structure de l'institution ou de la société et la cosmologie de la société sont rendues visibles autour de cette personne, par la façon dont on s'occupe d'elle. Pour moi, c'est ce qui fait le lien entre ces deux réalités.

[CL] - C'est d'autant plus vrai que, au Sri Lanka, vous vous intéressiez aux questions de santé mentale?

[LR] - Les Occidentaux ont effectivement tendance à dire que les rituels cingalais sont une sorte de soin psychologique, pour des problèmes qui relèvent de la psychologie. Mais, dans le village où je travaillais, les gens disaient qu'ils chassaient les démons qui provoquaient des troubles à la fois psychologiques et physiques. En réalité, il y avait un processus de sélection, et les maladies physiques avaient plus de chance d'être traitées par la biomédecine - par le biais de l'apothicaire du village ou par les hôpitaux accessibles -, tandis que les maladies psychologiques faisaient l'objet de rituels de guérison. Je dirais que le fil rouge, c'est la façon dont les gens se rassemblent autour d'un problème. Mais il y a par ailleurs un autre fil : le fait qu'à chaque fois, les choses se passent à l'intérieur d'un espace fermé. Bien sûr, ce type d'espace se prête mieux à l'ethnographie. Tout le monde est là, tout arrive dans un même endroit. C'est quelque chose qui m'a toujours fascinée, dès le moment où j'ai commencé à lire des choses sur les rituels de guérison. Les anthropologues décrivaient un espace - un intérieur - dans lequel une situation problématique se déroulait et où se déployait une tentative de s'organiser autour de cela, de faire quelque chose pour y répondre. Que ce soit dans un rituel de guérison, un hôpital psychiatrique ou une prison, on observe chaque fois la façon dont les personnes mobilisent les ressources culturelles dont elles disposent.

$[C L]$ - Du Sri Lanka à la psychiatrie, puis à la prison, on a le sentiment que vous vous êtes intéressée à des institutions de plus en plus coercitives : est-ce un hasard, ou est-ce que cela témoigne de votre intérêt croissant pour la coercition?

[LR] - Il me semble que c'est un hasard. D'une certaine façon, je n'ai fait que suivre ma trajectoire de vie, de travail, et il se trouve que j'ai eu la possibilité d'étudier ces lieux. Ce qui relève le plus du choix dans mon parcours, c'est d'être allée au Sri Lanka et d'avoir décidé d'étudier les rituels de guérison. C'est une décision que j'ai prise 
alors que j'étais à l'Université, et j'aurais pu choisir de faire autre chose. Mais après cela, une chose en a amené une autre. Vous avez bien sûr raison de relever le thème de la coercition. Je n'en avais pas vraiment conscience lorsque je suis passée de la situation au Sri Lanka à l'hôpital. Peut-être parce que le premier hôpital que j'ai fréquenté utilisait encore pour une part un modèle psychanalytique, ce qui déterminait la façon dont les patients étaient considérés. Certes, c'était coercitif, mais certains aspects de cette coercition ne m'apparaissaient pas, parce que je n'avais pas assez d'expérience. Des choses comme la case conference (la discussion de cas), où le patient était questionné de façon très intrusive... mais à ce moment-là, je n'étais pas en mesure d'y voir une forme de coercition. Et certains patients étaient vraiment très malades. J'avais devant moi des psychotiques, des gens très perturbés, délirants, qui auraient été incapables de vivre seuls en dehors de l'hôpital, et pour qui l'hôpital était un refuge. Et puis je suis passée de l'hôpital psychiatrique à la prison et, là, j'ai $\mathrm{vu}$ des prisonniers qui auparavant auraient été patients dans des hôpitaux psychiatriques, et que l'on détenait de façon extrêmement coercitive, au point que l'on en venait à souhaiter qu'ils puissent être accueillis dans des asiles. Progressivement, il est devenu évident que j'étudiais la coercition, mais au départ, ce n'était pas explicitement mon objet.

\section{Comme si elles étaient dans la même pièce}

[LV] - Votre écriture mêle étroitement description ethnographique et théorisation. Nous avions donc envie de vous demander comment vous travaillez, et par quel processus vous aboutissez à ces textes si singuliers.

[LR] - Vous voyez ces petits carnets à spirale ? J'en suis extrêmement dépendante, j'en ai toujours un sur moi : ils ne sont pas encombrants, on peut y noter quelque chose très rapidement et on peut en tourner les pages facilement, et les garder sur les genoux. Un avantage de l'ethnographie institutionnelle, c'est qu'il y a constamment des occasions d'aller à des réunions, ou de s'asseoir avec quelqu'un... Dans ces situations, il est normal d'avoir un carnet. Je ne m'en sépare donc jamais. Dans la prison, ça n'a jamais gêné personne, et parfois même, quelqu'un me demandait : «Et alors, il est où, ce carnet? » ou « Vous notez, hein? ». Il y a quelque chose d'un peu mystérieux là-dedans, mais les gens investissent votre carnet. J'imagine que vous en avez aussi fait l'expérience. J'ai bien enregistré parfois des entretiens (et dans mes recherches sur la prison, c'était un processus assez complexe, qui impliquait d'autres gens que moi), mais une bonne part du matériau de mes livres vient de mes carnets. Il m'arrive de taper mes notes, mais c'est rare. À mes yeux, les notes tapées ne sont pas aussi vivantes que les notes manuscrites, même si souvent je les annote après coup, ou si j'y fais des ajouts... Il y a quelque chose dans les carnets eux-mêmes qui m'aide vraiment. Lorsque je les reprends, je peux reconstruire le contexte.

J'ai aussi découvert que l'écriture me vient beaucoup plus facilement lorsque je commence par raconter ce qui m'a particulièrement frappée. Tout le monde ne procède pas ainsi, je le vois avec mes étudiants. Beaucoup écrivent à partir d'une liste des points à aborder, qu'ils détaillent au fur et à mesure. Mais après un certain nombre de tentatives, j'en suis arrivée à la conclusion que, pour que votre travail ait quelque chose de vivant, il vaut mieux commencer par les choses qui vous ont paru les plus notables au début de la recherche, et qui faisaient pour ainsi dire écho à la 
situation dans son ensemble. Par exemple, quand j'écrivais le livre sur la prison, j'avais des réticences à commencer par raconter la façon dont les gens jetaient des matières fécales par la fenêtre de leur cellule, et toute la question du corps dans la cellule. Ça me paraissait terrible de partir de là. Mais mes collègues et moi, nous étions tous d'accord pour dire que c'était ce qui nous avait le plus déconcertés et troublés lorsque nous avions découvert le système carcéral. C'est ce qui m'a poussée finalement à commencer par là.

Comment est-ce que j'incorpore la théorie à cela? Je dirais que je construis les deux séparément : d'un côté, je fais de l'ethnographie et je ne réfléchis pas vraiment à la théorie, et de l'autre je lis, mais sans trop penser à l'ethnographie. Puis, sans que je puisse vraiment expliquer comment, lorsque je me mets vraiment à écrire, je les associe, comme si elles étaient dans la même pièce, et qu'elles se parlaient... J'ai aussi des notes sur des livres, et je vais de l'un à l'autre : ça se fait un peu de soi-même c'est assez désorganisé !

Avec le temps, j'ai compris aussi qu'il n'y avait pas de nécessité à ce que tel théoricien vous influence plutôt qu'un autre. Lorsque j'ai commencé à travailler à l'hôpital psychiatrique, j'étais persuadée que les analyses de Kleinman - qui était une figure majeure de l'anthropologie médicale à l'époque - me seraient utiles pour décrire ce que je voyais. Je me souviens d'avoir essayé de faire entrer dans le même espace Kleinman et l'hôpital... Mais ça ne marchait absolument pas ! C'était à la fois frustrant et énervant. Et puis, à un certain moment, j'ai découvert Foucault, et cela a été une révélation: "C'est donc là que cette conversation peut exister!". À partir de ce moment, j'ai su comment lire et faire de l'ethnographie parallèlement. C'est un aspect particulièrement excitant de ce travail pour moi: le moment où surgit l'étincelle! Vous êtes au beau milieu d'un travail d'ethnographie, vous avez le sentiment de tâtonner dans l'obscurité... et alors la perspective théorique arrive pour vous aider.

D'ailleurs, s'il y a un conseil que je donnerais aux apprentis ethnographes, c'est de ne pas trop s'attacher à une perspective théorique, de ne pas supposer qu'elle s'appliquera forcément, avant de s'être immergé dans la recherche ethnographique. Ce n'est pas toujours évident, parce que les bourses et les postes d'enseignement vous poussent souvent à définir a priori la perspective que vous allez adopter. Mais un certain degré d'incertitude et d'ouverture est plus susceptible de conduire à un travail original. Donc, une chose que je pourrais dire, c'est: travaillez à fond la théorie, mais mettez-la ensuite de côté un moment, pour vous permettre d'être simplement dans l'endroit que vous étudiez. Il arrive qu'on renonce à une théorie qu'on appréciait au profit d'une autre : ne vous interdisez pas cette possibilité.

\section{Qui d'autre le fera?}

[LV] - Chacune de vos recherches a nécessité une immersion longue dans des terrains éprouvants. Rétrospectivement, comment définiriez-vous l'intérêt qui vous a guidée à travers vos différentes expériences ethnographiques et qui a permis de maintenir cet engagement?

[LR] - Je pense que c'était l'intérêt pour l'intérieur institutionnel et les relations qu'on peut voir s'y développer. C'est quelque chose qui m'a toujours fascinée. Une fois qu'on a commencé à faire de l'ethnographie dans une institution, les choses sont 
relativement faciles : il suffit d'y aller et d'y retourner, encore et encore. Le défi, c'est de parvenir à entrer, mais une fois que cela est acquis, vous vous retrouvez dans cette situation incroyable, où vous pouvez pénétrer dans ces espaces et avoir accès à des formes d'activité humaine réellement extraordinaires. Il y a tellement d'énergie émotionnelle accumulée dans un service psychiatrique ou dans une prison. Et aussi bien le personnel que les résidents semblent parler de leur vie et de leurs sentiments avec plus de liberté qu'ils ne pourraient le faire dehors. Cela m'a vraiment captivée. C'est un cliché de dire qu'on pénètre dans un "monde ", mais c'est pourtant vrai, et les habitants de ce monde semblent vouloir vous y faire entrer de plus en plus profondément. Ils veulent que vous compreniez. C'est ce qui donne au travail sa dynamique propre.

J'ai toujours été très motivée par l'écriture de mes livres. Notamment celui sur Baltimore (Emptying beds), lorsque l'équipe de l'hôpital m'a autorisée à entrer dans son monde, et que j'ai pris conscience que je pouvais le décrire. Mais c'était aussi le cas avec la prison... J'étais en train de conduire vers la prison... elle était située loin de la ville : à cette époque, je me levais à quatre ou cinq heures du matin pour être à la prison à huit heures. Je me souviens du moment exact où, alors que j'étais au volant de ma voiture, j'ai pris conscience que j'avais non seulement la possibilité d'aller dans ce lieu où il était si difficile d'entrer, mais aussi la formation théorique nécessaire pour en rendre compte... Il fallait écrire un livre, c'était une évidence. À partir de là, j'avais le sentiment d'être investie d'une mission. Pour le formuler simplement, ça se résumait sans doute à quelque chose comme: «Qui d'autre le fera?» Il y a bien Foucault, qui a décrit les choses au milieu desquelles je me trouve, mais qui d'autre est là, qui pourrait écrire là-dessus ? C'est quelque chose dont j'ai pris conscience d'un coup, cette obligation que j'avais de ne pas gâcher l'occasion qui m'était donnée... Il existait tellement peu de choses sur les prisons de très haute sécurité, les Supermax, mais c'était aussi le cas pour la situation psychiatrique : à l'époque, elle n'avait pas été très étudiée.

\section{Par la description, on rend possible la dénonciation}

[CL] - Au départ, vous étiez motivée par une certaine curiosité. À partir de quel moment avez-vous éprouvé le besoin de révéler, voire de dénoncer, ce que vous aviez observé en prison?

[LR] - Une chose importante était d'entrer à l'intérieur pour pouvoir dire : "Voici comment les choses se passent ». Un pays qui crée des lieux comme ceux-là, dont personne n'a conscience... ça ne devrait pas être un secret. Mais il y avait autre chose. Au départ, j'avais été vraiment frappée par la cohérence entre ce que disait Foucault de la prison et ce que je pouvais en voir moi-même. Mais, avec le temps, j'ai pris conscience que je percevais davantage la complexité de ce système, et c'est ce que je me suis appliqué à explorer, en pénétrant toujours plus profondément dans la réalité de la prison. J'ai vu certaines choses au début que je ne comprenais pas très bien, et c'est pour ça que c'était formidable de pouvoir y consacrer de nombreuses années. Quand on a du temps, on peut mieux connaître les gens et mieux comprendre comment les choses se passent. C'est ce que je voulais décrire. Quand j'ai commencé à m'intéresser à la psychiatrie, l'aspect de dénonciation ne m'apparaissait pas. Ça peut sembler naïf, mais à l'époque, dans la recherche, la dénonciation se cantonnait à un 
discours assez abstrait sur le «contrôle social » ou l'«asile». Cette violence ne me touchait pas autant que la situation extrême dont j'étais témoin tous les jours, avec des patients souffrant de graves pathologies mentales et souvent sans domicile. Ce n'était pas de façon évidente une situation qui exigeait d'être dénoncée. En revanche, en prison, n'importe qui aurait vu qu'il était crucial et urgent de dénoncer la situation. Quand j'ai découvert les quartiers d'isolement (solitary confinement facilities) et les prisons de très haute sécurité, les Supermax, où l'isolement des détenus est systématique, il était clair pour moi que j'avais la responsabilité de faire quelque chose et, par la description, on rend possible la dénonciation. Mais si l'on part de la dénonciation, on n'arrive pas au même résultat. Et puis bien sûr il y avait mes habitudes... Être ethnographe fait partie de ce que vous êtes, ce n'est pas quelque chose qu'on puisse séparer de soi. Et il n'est pas possible de faire un travail d'ethnographe si dès le départ on veut absolument dénoncer quelque chose. À mes yeux, la dénonciation et l'activisme sont extrêmement précieux face aux prisons et aux autres institutions coercitives, mais le chemin que j'ai pris est différent.

[LV] - Mais alors, que faites-vous de la position critique de Foucault, ou de la façon dont on peut l'utiliser comme outil de dénonciation ? Pourquoi reste-t-il si utile pour vous?

[LR] - Est-ce que vous ne pensez pas que c'est aussi précisément ce que fait Foucault ? Foucault décrit des situations, des systèmes, et il sait que nous allons voir où se situe le problème. Il le montre de façon si incroyablement évidente qu'il serait inutile qu'il ajoute : «Regardez, il y a un problème!» J'appréciais beaucoup ses travaux, ses analyses étaient tellement cohérentes avec ce que je pouvais observer. C'était déjà vrai à l'hôpital psychiatrique, mais plus encore en prison ${ }^{6}$. L'une des raisons pour lesquelles je n'ai pas rencontré Goffman comme j'ai rencontré Foucault est que ce que décrivait Goffman appartenait déjà au passé lorsque j'ai découvert l'hôpital psychiatrique. Bien sûr, ses descriptions sont, elles aussi, exactes et utiles, mais elles décrivent une situation antérieure à celle dans laquelle je me trouvais. Pour une part, c'était donc une question de temporalité. Si j'avais travaillé dans les années 1960, j'aurais pu vivre la même chose avec Goffman.

\section{Une responsabilité envers la complexité}

[CL] - Dans vos livres, vous révélez ces mondes fermés et vous en montrez la réalité ambivalente: rien n'est jamais tout blanc ni tout noir. Comment parvenez-vous à saisir cette complexité et à l'expliquer simplement?

[LR] - Pour moi, la complexité est une évidence. On ne peut pas y échapper. Il n'est pas possible de comprendre un endroit quand on vient d'y arriver. C'est une forme d'incompréhension que l'on pourrait comparer à l'apprentissage d'une langue étrangère, même si l'incompréhension est plus subtile. En un sens, tout est transparent : les gens vous parlent, vous savez ce dont ils parlent, vous partagez plus ou moins le même univers culturel... et pourtant, on sent immédiatement des strates qui nous échappent dans ce qui se passe. J'ai l'impression d'en avoir toujours eu très clairement conscience. À partir de là, mon travail a simplement consisté à suivre différents fils à la fois. Quelqu'un me parle de quelque chose, je le note dans mon petit carnet, et ensuite quelqu'un me dit autre chose, et plus tard je reviendrai peutêtre leur reposer la question. Il n'y a là rien d'autre que la méthode ethnographique 
classique : les gens vous livrent eux-mêmes la complexité, ils veulent que vous la connaissiez. Pour moi, c'est cela, l'ethnographie, c'est ce processus.

[CL] - Et pourtant, tout ethnographe désire tisser tous ces fils en un seul, tandis que vous résistez à cette impulsion dans vos ouvrages : comment faites-vous?

[LR] - C'est vrai, j'y résiste. Bien sûr, je suis convaincue qu'il n'y a aucune bonne raison de maintenir des gens à l'isolement plus de quelques jours. À ce niveau-là, il y a une idée unificatrice. Mais cela ne vous aide pas à comprendre pourquoi on y garde des gens pendant des années. Et par ailleurs, il y a des situations plus ambiguës que celle-là. Quelle est la bonne chose à faire, quelle est l'attitude juste face à quelqu'un qui est psychotique et incapable de prendre soin de lui-même ? Il est clair que ce que nous faisons ne marche pas terriblement bien, mais qu'est-ce qui serait préférable? Tout cela est très obscur, et c'est une question avec laquelle les gens se débattent depuis des générations.

[LV] - Je suis très sensible à cette idée que la complexité serait au cœur de l'ethnographie. En même temps, il est délicat de réussir à exprimer cette complexité sans se contenter de dire que tout est complexe. Votre style, justement, a une façon très personnelle de tisser narration et explication, qui parvient à trouver un équilibre subtil.

[LR] - Merci ! Je vois ce que vous voulez dire. Les ethnographies qui se réduisent à une grande description complexe et impénétrable ne fonctionnent pas. L'auteur a une responsabilité envers la complexité, il doit quelque chose à la situation qu'il décrit, mais il a simultanément une responsabilité envers la personne qui va le lire. $\mathrm{Au}$ lecteur, on doit une certaine clarté et des éléments d'orientation. L'auteur doit créer des espaces de respiration, où le lecteur puisse prendre un peu de distance et comprendre ce qui se passe. Quand je suis immergée dans la recherche, j'ai du mal à écrire, parce que je ne vois pas ce qui se passe et que je serais bien en peine de guider quelqu'un d'autre. Mais plus tard, lorsque je me mets à y réfléchir devant ma page, je me demande ce dont le lecteur a besoin pour comprendre. Il me semble que c'est comme ça que s'est construit ce style d'écriture.

\section{Les institutions ne cessent de promettre et de surenchérir sur leurs promesses}

[CL] - Vous semblez assez pessimiste quant à l'avenir des institutions fermées. C'est en tout cas ce qui ressort à la fois de Total Confinement et de votre intervention sur la répétition, l'entropie et l'intérieur institutionnel à la conférence "L'enfermement au prisme des sciences sociales: rapprocher les lieux, confronter les approches » à Bordeaux en octobre $2013^{7}$.

[LR] - C'est vrai, mon travail est assez pessimiste concernant les institutions. Il est possible que nous n'en finissions jamais avec elles, et qu'elles perdurent indéfiniment sous une forme ou une autre, mais elles paraissent en même temps rencontrer de façon récurrente des limites à la fois éthiques et pratiques. Il me semble que c'est particulièrement le cas de la prison, mais on peut sans doute dire la même chose de la psychiatrie aux États-Unis: on a tenté de créer une psychiatrie communautaire vivante, mais les résultats n'ont pas été à la mesure des promesses... Donc, oui, les institutions ne cessent de promettre et de surenchérir sur leurs promesses, et finalement... cela ne fonctionne pas, et elles font de nouvelles promesses ! C'est ce que j'évoquais dans cette communication. Je voulais faire sentir comment elles se 
défont, puis se reconstituent en prenant une autre direction... la solution adoptée ne répondant jamais réellement au problème qu'elles tentent de résoudre. Peut-être à cause de cela, les recherches sur les institutions ont elles aussi tendance à se répéter, à être prévisibles. Je pense ici aux institutions fermées ; la réponse à cette question se trouve sans doute en dehors de cet espace fermé... Ou, pour le dire autrement, quand on se demande ce qui doit arriver ensuite ou comment le penser, la fermeture ne fait pas nécessairement partie de la réponse.

[CL] - Vous avez étudié à la fois le monde de la psychiatrie et celui de la justice : pensezvous que ces institutions connaissent des évolutions communes?

[LR] - Je dois dire que je suis assez inquiète de la direction générale qu'elles prennent, $\mathrm{du}$ moins au États-Unis. Beaucoup d'efforts sont faits pour créer des unités psychiatriques à l'intérieur du système carcéral. C'est un énorme investissement en argent et en personnel que de préserver les personnes souffrant de troubles psychiques de l'environnement carcéral ordinaire... Mais de telles solutions sont fragiles, même si elles peuvent paraître fermement établies pour un temps. En fait, il est difficile d'imaginer qu'elles puissent être maintenues longtemps... Si vous reprenez la description que donne Foucault de l'hôpital général - cette première institution, où tout le monde était détenu de façon indistincte -, finalement, c'est la solution la moins coûteuse. Et dans une certaine mesure, le système carcéral américain en vient à ressembler à cet hôpital général. Dans de nombreux cas, il se contente d'absorber les patients psychiatriques plutôt que de créer un parcours psychiatrique fonctionnel distinct... C'est là une réponse possible à votre question. Mais il y a aussi des indices de changement positif aux États-Unis. La mise à l'isolement des détenus est moins invisible qu'elle ne l'a été et elle fait aujourd'hui l'objet de critiques publiques. Par ailleurs, du fait de la complexité du système américain, il peut y avoir des initiatives locales encourageantes : l'État de Washington travaille ainsi à réduire certains des problèmes des détenus souffrant de pathologies psychiques, et ceux posés par la mise à l'isolement. La rhétorique du « tough on crime " (le slogan popularisé par Reagan, qui préconisait d'être "dur contre le crime ", d'avoir une politique particulièrement répressive pour dissuader les criminels) s'est aussi un peu essoufflée au niveau national.

\section{Au risque d'être naïve}

[CL] - II y a quand même une note d'optimisme dans le court article que vous avez écrit sur la prison de Grendon (Rhodes, 2010)ㄹ, en Angleterre. Lorsque vous l'avez visitée, vous avez été profondément étonnée, n'est-ce pas ?

[LR] - C'est vrai : j'ai eu la surprise de découvrir une institution qui parvenait à mieux gérer les prisonniers les plus violents. Visiter Grendon m'a réjouie à plus d'un titre comme cela a réjoui de nombreux chercheurs au fil des ans. C'était d'autant plus important pour moi d'être là que j'avais travaillé dans des environnements extrêmement oppressifs. Grendon démontre que des individus qui ont une histoire de violence et de traumatismes sont capables de changer lorsqu'ils se retrouvent dans un environnement positif et riche. 
[CL] - Dans cet article, on a le sentiment que c'est au public américain que vous vous adressez. Son titre - «Risking Therapy » («Prendre le risque de la thérapie ») - n'est-il pas une façon d'interpeller l'administration pénitentiaire américaine?

[LR] - Oui, tout à fait : c'est avec cette perspective que j'ai réfléchi à cet article et que je l'ai écrit. Le risque est une telle obsession dans le système carcéral ici et, d'ailleurs, cette obsession transparaît aussi d'une certaine façon à Grendon. Mais le fait qu'ils aient tenté cette approche depuis tant d'années me paraît très significatif... Je ne pense pas qu'un article comme le mien puisse avoir le moindre effet sur les orientations de l'administration pénitentiaire dans ce pays, mais il est néanmoins porteur d'un message pour qui veut bien l'écouter.

Une autre chose qu'il faut noter : il y a un vrai risque lorsqu'on écrit sur des lieux comme celui-là, on peut facilement avoir l'air naif, parce qu'il s'y produit des choses profondément touchantes et séduisantes. C'est quelque chose dont il est très difficile de parler au-dehors, parce qu'on n'a pas envie de donner l'impression qu'on oublierait la dimension coercitive de ces lieux. Il faut critiquer la coercition, mais, à l'intérieur de ce cadre, tellement de choses peuvent arriver. Certaines sont positives, même si l'on pourrait dire qu'en un sens elles le sont de façon perverse! Dans les hôpitaux psychiatriques et dans les prisons, les gens nouent des relations amoureuses, ils plaisantent, ils tiennent des journaux intimes, ils éprouvent de la compassion, de la haine ou de la tristesse... C'est ce qui est tellement fascinant. Et là encore, si l'on ne raconte qu'un côté de l'histoire pour mieux dénoncer, on manque toutes sortes de choses.

[LV] - Ce que vous suggérez aussi, c'est que, dans ces institutions, on touche le cœur de ce qui fait une société, et que c'est toujours quelque chose d'ambivalent.

[LR] - C'est effectivement mon expérience. La chance de l'ethnographe, c'est que, lorsque les gens s'aperçoivent que vous prêtez attention à cette ambiguïté et à cette complexité, ils veulent que vous y fassiez encore plus attention. Vos enquêtés se mettent à participer à votre travail d'ethnographie, et c'est ce qui fait se multiplier les fils. À mes yeux, c'est là la condition de possibilité de ce travail.

\section{L'ethnographie rend heureux}

[CL] - Vous avez pris votre retraite récemment, mais nous vous proposons un exercice d'imagination: si vous deviez consacrer dix ans à une nouvelle recherche, comment l'envisageriez-vous?

[LR] - L'idée de commencer quelque chose de tout à fait différent est parfois attirante. Une collègue de l'université de Washington étudie la politique de l'alimentation. Par bien des aspects, la question est déprimante, mais c'est aussi un sujet ouvert ; on enquête à l'extérieur et dans des endroits très divers, les débats sont vifs, les enjeux se renouvellent. On peut facilement imaginer poursuivre ce type de recherche tout en étant à la retraite. À l'inverse, réaliser une recherche à long terme dans des institutions totales est lourd émotionnellement et, à certains égards, physiquement, et la perspective d'entamer un nouveau travail de terrain m'effraie un peu. Je dois aussi reconnaître que j'apprécie d'être à la retraite et que je profite pleinement de la possibilité de m'intéresser à d'autres choses. Je n'ai eu aucun mal à déplacer mes centres d'attention - c'est peut-être aussi une affaire de génération et de genre. 
[LV] - Mais pensez-vous sincèrement que vous auriez trouvé suffisamment d'intérêt ethnographique à travailler sur une question aussi ouverte que celle de la politique de l'alimentation? Comme vous l'avez souligné, les espaces fermés auxquels vous avez consacré votre travail créent une intensité émotionnelle: les relations qu'on y développe sont différentes, on y est confronté à un réseau complexe de relations intenses... il me semble que c'est ce qui fait que le travail de terrain en vaut la peine?

[LR] - Quelle merveilleuse question! Il y a des années de cela, lorsque j'étais en postdoc à Harvard, après mon retour du Sri Lanka, j'ai commencé à mener des entretiens avec des femmes d'origine indienne. Dans les années 1990, j'ai aussi mené des entretiens pour des projets dans le domaine de la santé publique. Mais je n'y trouvais pas ce que j'apprécie dans les situations sociales fermées ou limitées. On n'y trouve pas ces fils que l'on peut suivre, parce que les différentes personnes qui vous parlent n'ont pas de lien entre elles. Bien sûr, on peut ensuite discerner suffisamment de similitudes entre elles pour rendre le tout cohérent, mais ça n'a rien à voir avec des gens qui vivent ensemble tous les jours. J'imagine qu'à l'époque où les anthropologues se rendaient dans des lieux où subsistaient encore de petites communautés, c'était plus facile, et peut-être plus fort d'une certaine façon. On peut peut-être avancer l'hypothèse que ce qui nous plaît dans les institutions fermées, c'est quelque chose que nous avons perdu dans notre société. L'intérieur d'une institution est une sorte de version perverse de la société : comme d'autres l'ont relevé, l'institution peut constituer comme une "petite société », et c'est quelque chose qui, par certains côtés, a la préférence de l'ethnographie.

Mais je voudrais conclure sur une note plus joyeuse. Ce que j'ai dit de ma retraite pourrait laisser penser que mon travail était déprimant. Mais si je considère globalement mon expérience d'ethnographe, je dirais que faire de l'ethnographie vous rend heureux. Même lorsqu'elle confronte à l'insoutenable, c'est une forme de travail extraordinairement gratifiante.

\section{Extrait de l'ouvrage Total Confinement, Madness and Reason in the Maximum Security Prison (Rhodes, 2004, 139-148) :}

5 Cet extrait est tiré de la seconde partie de l'ouvrage, intitulée « Négocier le traitement, gérer la détention » (« Negotiating treatment, managing custody»), dans laquelle Lorna Rhodes s'intéresse aux jugements opérés par les professionnel.le.s présent.e.s dans les unités de haute sécurité (Supermax) pour évaluer et répondre aux comportements des personnes détenues qui semblent présenter des troubles psychiques. La question posée dans cette partie est celle de savoir ce qui détermine la décision de considérer les individus comme malades ou non, et donc comme relevant d'une prise en charge médicale ou comportementale. Dans cet extrait, Lorna Rhodes suggère que cette opération de tri opéré par les professionnel.le.s repose fortement sur leur compréhension de l'outil de classification des troubles psychiques de l'Association américaine de psychiatrie (APA) : le Manuel diagnostique et statistique des troubles mentaux (Diagnostic and Statistical Manual of Mental Disorders ou DSM ${ }^{9}$ ). 


\title{
Des troubles mentaux clairs et distincts
}

\author{
«Le processus actuel de révision du DSM donne \\ une impression de précision et d'exactitude. Et \\ effectivement, nombreux sont ceux qui en sont \\ arrivés à croire que nous avons affaire à des \\ troubles clairs et distincts, plutôt qu'à des \\ assemblages arbitraires de symptômes. " \\ (Tucker, 1998, 159) \\ « La prison est un jardin botanique du DSM. » \\ (Un travailleur en santé mentale) \\ « Schizophrène, schizoïde... » \\ (Un prisonnier se décrivant lui-même)
}

6 Depuis le XIX ${ }^{e}$ siècle, le contrôle, la réhabilitation et la psychiatrie sont profondément entremêlés - bien qu'en des proportions variables (voir par exemple Roberts, Gebotys, 2001 ; Rothman, 1998 ; Rotman, 1998). Le sociologue John Irwin décrit les effets du basculement des grandes orientations politiques qui eut lieu à partir des années 1950, alors qu'il était détenu à Soledad. À ce moment-là, de nombreux établissements pénitentiaires adoptèrent un modèle médical optimiste de la criminalité et furent rebaptisés «corrections» («maisons de correction», ou de « redressement »). Au début des années 1960, «l'ère du traitement était accueillie avec enthousiasme [...]. Les détenus [...] étaient amenés à croire qu'ils pourraient accroitre leur niveau de formation [...], apprendre un métier [...] et recevoir de l'aide [pour résoudre] leurs problèmes psychologiques. » Cependant, dans les années 1970, les prisonniers commencèrent à suspecter qu'il s'agissait là d'« une vaste hypocrisie visant à camoufler sous le nom de "traitement" des exigences administratives, des préoccupations liées à la détention et à la surveillance et une approche punitive. » (Irwin, 1970, 52-53, voir aussi Irwin, 1980 ; Wicker, 1975). Dans les années 1980, l'accent fut de nouveau mis sur la neutralisation et la punition, considérées comme les réponses les plus rationnelles à la criminalité. En 1997, évoquant l'extraction d'un détenu d'une cellule d'un quartier de haute sécurité, John Irwin et James Austin déploraient que «la réhabilitation, le principe fondateur de la pénologie, [soit] tombée en disgrâce. » (Austin, Irwin, 2001, 97)

C'est seulement à la lumière de cette histoire que l'on peut comprendre la façon dont le traitement, auparavant conçu comme un projet global de « redressement» (corrections), est aujourd'hui réduit à la "santé mentale $»^{10}$. Selon cette acception plus étroite, le traitement requiert que les intervenants en santé mentale contrôlent l'accès à leurs lits, en nombre limité, en s'appuyant avant tout sur la classification psychiatrique établie. Le DSM-IV (la quatrième édition du Manuel diagnostique et statistique des troubles mentaux) se trouve en bonne place sur le bureau de tous les psychiatres et professionnels en santé mentale. $\mathrm{Au} v \mathrm{vu}$ du nombre considérable de personnes concernées par l'incarcération aujourd'hui, la principale vertu de cette bible de la psychiatrie est de permettre de distinguer d'une façon semble-t-il assurée les fous des personnes simplement nuisibles. [...] Pour diagnostiquer la schizophrénie, un travailleur en santé mentale peut recourir à l'arbre décisionnel du manuel afin de vérifier la présence de certains groupes de symptômes: "La schizophrénie est une affection qui dure au moins 6 mois et inclut [...] deux (au moins) des manifestations suivantes: idées délirantes, hallucinations, discours désorganisés [...]» (American Psychiatric Association, 2000, 344) ${ }^{11}$. 
8 J'ai eu un jour l'occasion de suivre un détenu, Eddie Mullen, lors de son admission au sein d'une unité de soins psychiatriques ${ }^{12}$. Il avait été incarcéré peu de temps auparavant après avoir agressé, en état d'ébriété, des membres de sa famille. Il était petit, débraillé, et portait plusieurs tatouages et cicatrices. Le travailleur en santé mentale chargé de son admission l'interrogea avec soin au sujet de son crime et de ses symptômes. Mullen se décrivait comme ayant «mal à l'intérieur » et comme souffrant de paranoïa et d'angoisse. «Des fois, j'entends des trucs qui ne sont pas là, des trucs qui ne sont pas très clairs [...]. Je m'évanouis tellement je suis angoissé - des crises d'angoisse, c'est comme ça qu'elles sont classées. L'an dernier, j'ai voulu me faire sauter la cervelle, mais j'ai paniqué, je me suis dégonflé. » Il exprimait des remords, pleurait et se demandait s'il pourrait se « pardonner » ce qu'il avait fait.

9 Le travailleur en santé mentale l'écoutait attentivement. Il suggéra avec douceur à Mullen de faire du sport dans la cour de la prison, de se doucher régulièrement et d'entreprendre un programme d'accompagnement pour traiter son agressivité et sa dépendance à l'alcool. L'essentiel, lui dit-il, est d'« arriver à vous contrôler ». Mullen était d'accord : «C'est pour ça que je suis ici, pour apprendre les bases. » Une fois qu'on eut retiré ses menottes à Mullen et qu'il eut été escorté jusqu'à sa cellule par un gardien, le travailleur en santé mentale se tourna vers moi :

Je penche pour un trouble de la personnalité. Ses tatouages suggèrent qu'il est antisocial, nous trouverons peut-être une bonne dose de colère. Par ailleurs, il faudrait pouvoir écarter le diagnostic d'état-limite (borderline) qui est suggéré par le fait qu'il donne des coups contre les murs [...]. Ça évoque un peu un état-limite. Il entend parfois des voix, mais il n'est pas schizophrène. Il est très émotif, il a peutêtre un trouble anxieux, mais, pour moi, il s'agirait plutôt d'un trouble secondaire. Qu'est-ce que ses remords peuvent [vraiment] vouloir dire?

10 Ces commentaires - et l'ensemble de la conversation avec Mullen - manifestent l'usage fait au quotidien des catégories du DSM, ainsi que les présupposés sur lesquels celles-ci reposent. Mullen avait intérêt à se présenter comme souffrant d'une grave pathologie psychiatrique parce qu'il craignait d'être envoyé dans un environnement plus dangereux ${ }^{13}$. Il se décrivait ainsi comme paranoïaque, angoissé, délirant et rongé par le remords. Les doutes du travailleur en santé mentale s'exprimaient quant à eux à travers différentes catégories diagnostiques : trouble de la personnalité antisociale ou état-limite. Il cherchait des indices au-delà de ce que Mullen pouvait dire de lui-même, dans sa colère, ses tatouages et ses cicatrices. Il ne croyait pas aux remords que celui-ci pouvait exprimer.

11 Les définitions diagnostiques du DSM ne font pas référence à des personnes individuelles, à leur histoire ou même à leur personnalité. Au lieu de cela, elles fournissent un langage permettant de décrire des ensembles de caractéristiques qui devraient être claires pour tout observateur bien formé ${ }^{14}$. Les troubles sont divisés en plusieurs axes, vastes catégories taxonomiques qui distinguent les maladies (ou «états») et la personnalité (ou «traits»). L'Axe I concerne les syndromes cliniques et inclut les principales maladies mentales que sont la schizophrénie, la dépression et les troubles bipolaires. Dans le langage courant employé en psychiatrie, cet axe concerne les «fous » dont les symptômes sont reconnaissables et souvent assez exubérants, mais pour lesquels il existe, en général, des traitements (presque toujours pharmacologiques). L'Axe II renvoie à des troubles de la personnalité (et à certains troubles du développement). Il englobe l'« anxieux », l'« excentrique », le « capricieux » 
- ceux dont les traits de caractère sont issus de et aboutissent à « un conflit entre l'individu et la société » (APA, 2000, 789-790 ; Lowe, 1995, 157) ${ }^{15}$.

Par ses propos, Mullen suggère un diagnostic relevant de l'Axe I. Si un diagnostic de schizophrénie paranoïaque ou de trouble anxieux était posé, son comportement pourrait être envisagé comme symptomatique, ce qui porterait à envisager un traitement médicamenteux. Dans ce cas, il aurait une maladie ${ }^{16}$. Mais si, comme le suggère l'intervenant en santé mentale, il devait être diagnostiqué comme relevant de l'Axe II, cela signifierait que ses troubles seraient considérés comme relevant du " caractère ". Son comportement antisocial serait perçu comme un trait enraciné dans sa personnalité, non susceptible d'être modifié par un traitement médicamenteux ou autre. Il serait « comportemental ».

13 Le diagnostic psychiatrique est l'un des mécanismes fondamentaux par lesquels les intervenants en santé mentale négocient l'acceptation ou le refus de ceux qui leur sont adressés. De leur point de vue, le diagnostic d'un trouble mental grave signifie que le prisonnier peut être aidé par ce qu'ils ont à offrir, notamment des traitements médicamenteux. Le prisonnier de l'unité de haute sécurité qui, au chapitre précédent, disait que les intervenants en santé mentale "vous souhaitent [un traitement médicamenteux] » touchait juste sur un point: «souhaiter » un traitement, c'est souhaiter qu'il y ait un traitement. Dans la logique circulaire de la psychiatrie biologique, quand les antipsychotiques et les antidépresseurs sont efficaces, c'est que le prisonnier est psychotique ou en dépression. Il est donc pertinent de parler de cercle, puisque la maladie du patient est identifiée par ce qu'il dit de lui-même, directement ou à travers certains indices. Dans les notes cliniques prises à propos de cas, on constate que, lorsque la personne est diagnostiquée comme relevant de l'Axe I, on trouve des spéculations quant aux conséquences de traumatismes passés, des considérations sur les délires et les idées paranoïaques et des récits de tentatives de suicide et de difficultés à prendre soin de soi. Un travailleur en santé mentale déclare ainsi : « Notre pouvoir consiste à approcher la personne en présupposant qu'il est possible de la changer ». Autrement dit, ce dont souffre la personne relève d'un état.

Comment établit-on la différence entre un trait de personnalité et un état? Les éléments de diagnostic - comme la cohérence du discours de Mullen et le fait qu'il exprime des remords - jouent un rôle important dans l'équation. Mais lorsque le travailleur en santé mentale déclare : "Ça évoque un peu un état-limite ", il considère également sa propre réaction émotionnelle comme un indice pertinent pour établir un diagnostic. Évoquant la formation des jeunes psychiatres, Luhrmann écrit, à propos de la distinction entre Axe I et II : la formule " "ça sent l'Axe II" correspond moins à une catégorie diagnostique bien définie qu'à une idée générale du trouble de la personnalité, avec tout ce qu'elle véhicule de malaise et d'irritation ». Pour beaucoup de psychiatres qui travaillent en dehors des prisons, « les patients atteints de troubles de la personnalité sont ceux qu'on n'aime pas, à qui on ne fait pas confiance, dont on voudrait se débarrasser [...]. L'une des raisons de cette aversion est le sentiment profondément ancré qu'ils sont moralement coupables, parce qu'ils auraient pu choisir d'être autrement. » (Luhrmann, 2000, 14, 115). À l'intérieur des prisons, le problème n'est pas de savoir si les psychiatres eux-mêmes «croient » à la distinction entre Axe I et II : ils sont en effet nombreux à avoir une perspective tout à fait nuancée sur ses usages et ses limites. L'enjeu réside plutôt dans la façon dont les professionnels de santé et plus généralement le personnel pénitentiaire utilisent la clarté apparente de ce 
système de classification dans leur travail quotidien pour classifier les prisonniers et interagir avec eux.

L'entretien avec Mullen nous suggère l'importance que peut avoir la classification diagnostique dans le contexte pénitentiaire. La méthode simple que propose le DSM pour distinguer «maladie » et « comportement » sous-tend toute la conversation pour le travailleur en santé mentale, mais aussi pour Mullen, dans la mesure où lui aussi utilise le vocabulaire de la psychiatrie. Tous deux s'affrontent quant à la découverte et la définition de la "vérité " le concernant, qui constitue un enjeu pour l'un comme pour l'autre. Le travailleur de santé s'inquiète de la possibilité de manquer un diagnostic de personnalité anti-sociale, le diagnostic d'Axe II, le plus courant en prison, qui est globalement synonyme de criminalité masculine. Il craint en effet, si c'était le cas, que Mullen ne mette en danger les détenus les plus vulnérables, voire sa propre équipe. Du point de vue de Mullen, être diagnostiqué antisocial reviendrait à voir se fermer la possibilité d'être traité comme quelqu'un qui a souffert plutôt que comme quelqu'un de mauvais. S'il était une plante, il attendrait passivement d'être classé à l'entrée du fascinant jardin botanique du DSM qu'est la prison, aux yeux du travailleur en santé mentale cité en ouverture de ce texte. Mais comme c'est un être humain et qu'il parle, Mullen s'efforce de participer au processus qui doit aboutir à sa classification. Le problème est que ses mots, dans la mesure où ils n'expriment pas nécessairement la vérité sur lui, peuvent révéler une autre vérité, à savoir qu'il se livre à une manipulation. Dans ce cas aussi, une place lui revient : ce ne sera pas le lieu clos $\mathrm{du}$ traitement, mais la route principale, celle vers laquelle sont orientées les personnalités antisociales.

\section{Il est toujours sur le point de faire l'idiot (get stupid)}

« Parfois, j'en ai ras le bol qu'ils soient tellement idiots. L'autre jour, j’ai hurlé [sur un détenu],

c'est sorti tout seul.»

(Un gardien d'une unité de santé mentale)

« Être rationnel signifie ne pas mettre en question une situation irrationnelle, mais en tirer profit autant que possible du point de vue de ses intérêts particuliers. »

(Adorno, 1994, 43)

"Chacun fait son propre nid. Si vous voulez vivre dans les plumes et le duvet, ce sera agréable. Si vous y mettez des galets, ce sera tout de suite moins confortable [...]. Et ça, ça vaut [...] aussi bien pour le personnel que pour les détenus. »

(Un gardien)

La classification et le DSM sont utilisés dans les situations où doivent être prises des décisions explicites concernant le placement. Mais qu'en est-il lorsque le détenu moyen s'attire des ennuis? La relation entre incarcération et traitement est sous-tendue par une logique vernaculaire qui permet de gérer les inconduites quotidiennes. Cette logique fait primer des formes d'expertise carcérale qui n'exigent pas de se prononcer sur ce qu'est le détenu (sur son diagnostic) mais s'attachent plutôt à ce qu'il fait. 
17 «Faire l'idiot » signifie se conduire mal ou de manière irrationnelle, mais d'une façon qui n'exige pas l'assignation à une catégorie. Le détenu «idiot » est celui qui est jugé capable de choix rationnels, mais n'exerce pas cette capacité. Cette idée est omniprésente en prison, et elle n'a pas le sens qu'on lui donne couramment à l'extérieur. C'est ce dont j'ai pris conscience pour la première fois lors d'une conversation avec un adolescent dans une unité de santé mentale. Avec son air doux et ses cheveux bouclés qui formaient comme un halo autour de sa tête, il avait été victime d'une violente attaque dans la cellule qu'il partageait avec quatre autres détenus en régime ordinaire. "La prison, c'est dur à mon âge, me dit-il, beaucoup de gens s'attaquent aux jeunes. [Certains] gardiens cherchent à te faire enrager et à t'attirer des ennuis. Il y a beaucoup de gens dont il faut se méfier. Mais ici, on est plutôt en sécurité.» Il me raconta comment les détenus en régime ordinaire avec qui il se trouvait auparavant cherchaient à le convaincre d'aller tuer un autre détenu de l'unité où il était à présent. Certains n'avaient, disait-il, " aucune morale ». Alors que nous étions en train de parler, un gardien entra dans le bureau et il entendit sa dernière remarque. S'adressant à moi, il me dit : « Il est toujours sur le point de faire l'idiot », ce à quoi le garçon réagit en approuvant avec enthousiasme : « Ça m'arrive de faire l'idiot. Ce qui m'aide c'est [ce gardien qui se préoccupe de lui], les gardiens qui me crient dessus et mes amis [à l'intérieur]. Ils me disent quand je fais des conneries. Il y a tellement d'occasions de s'attirer des ennuis. »

Dans un contexte de pressions et de tentations multiples, son discours évoque les difficultés à s'auto-contrôler au quotidien et, face à cela, la possibilité d'une intervention immédiate, locale, d'un tiers. Plus tard, après avoir réentendu cette formule de «faire l'idiot » dans d'autres contextes, j'ai demandé à un gardien de m'en préciser le sens.

[Le gardien] - Faire l'idiot, ça veut dire en gros faire quelque chose qu'ils ne font pas [normalement]. Ça va être : ils sont escortés et ils se retournent contre le gardien sans raison, juste ils font l'idiot et ils sont punis pour ça.

[Lorna A. Rhodes] - Donc, quand les gardiens disent que quelqu'un a fait l'idiot, ça veut dire qu'il a explosé sans raison?

[Le gardien] - Oui, explosé sans raison... [Un détenu] essaie de frapper l'agent d'audience, de l'autre côté de la table. Ou il est escorté quelque part et il se retourne contre un gardien, des choses comme ça.

[LAR] - Donc, ce n'est pas de la connerie au sens de...

[Le gardien] - Non, faire l'idiot ce n'est pas être bête. Rien à voir.

[LAR] - Ça veut dire faire quelque chose sans...

[Le gardien] - Sans réelle justification.

[LAR] - Et si quelqu'un fait quelque chose comme ça, mais explique ensuite : «En fait, si je l'ai fait, c'est parce qu'untel m'a manqué de respect», ce serait encore faire l'idiot?

[Le gardien] - Ça dépend de quoi on parle. S'il l'a fait parce qu'un gardien lui a fait quelque chose, mais qu'il s'est attaqué à un autre gardien, ça resterait assez [idiot]. Mais s'il l'a fait parce que ce gardien lui a fait quelque chose pendant qu'il l'escortait, ça dépend. Le gardien va quand même considérer que c'est faire l'idiot. Mais pour le détenu, c'est justifié.

Il s'agit donc d'un concept de part en part social : il ne qualifie pas un prisonnier seul dans sa cellule, mais concerne une conduite sociale apparemment absurde ou peu réfléchie ${ }^{17}$. Le détenu affronte quelque chose qu'il n'a aucune chance de vaincre et il néglige les conséquences de son acte. De plus, la qualification d'un acte comme « idiot » 
dépend de ce qu'il a ou non des justifications, et ces justifications peuvent dépendre de la personne qui décrit les faits.

«Faire l'idiot» peut s'appliquer aussi bien à soi-même qu'aux autres, aux détenus comme au personnel. Un détenu, Sam Delano, déclarait ainsi avec mépris à propos d'un ancien compagnon de cellule :

Il a tué un type pour une histoire de drogue. Pour onze dollars de poison. C'était vraiment idiot. Si on vous arnaque, si on vous manque de respect, d'accord, défonce-lui la tête. Apprends-lui la vie. Mais ne le tue pas, ou en tout cas ne te fais pas prendre, hein. Si tu te fais prendre, alors la justice va t'envoyer ici, c'est triste, hein!

21 Un gardien d'une unité de haute sécurité parlait quant à lui de la frustration qu'il ressentait face à ces jeunes gardiens qui paraissaient ne pas avoir conscience des conséquences de leur conduite avec les détenus.

Toute action a des conséquences. Si je fais l'idiot à la grille, si je la secoue pour décrocher les détenus, si je fais l'idiot à la cantine, si j'humilie un type, il doit défendre son honneur devant ses pairs. Qu'est-ce que ça veut dire d'aller mêler à ça un autre membre du personnel, et de le faire tomber avec moi dans le trou que j'ai moi-même creusé ? Faut jamais créer une situation qui n'a pas besoin d'être créée.

Créer une situation qui n'a pas besoin d'être créée : voilà l'essence de ce que c'est que de « faire l'idiot ». Delano est un chaud partisan de l'usage de la violence, mais pour lui le meurtre - ou du moins se faire prendre à le commettre - est idiot. Dans ses remontrances à l'adresse de ses jeunes confrères, le gardien met en avant le contexte de respect et de mise en scène de soi au sein duquel de telles actions non nécessaires produisent leurs conséquences. Tel prisonnier dit encore : «Je savais qu'ils allaient me prendre ma radio, alors je l'ai défoncée et j'ai menacé [les gardiens]. J'ai juste fait l'idiot."

Parmi les détenus et le personnel, certains replacent ces conduites dans un contexte social plus global d'aliénation et d'absence d'opportunités. À propos des jeunes détenus de son unité, un membre du personnel déclare ainsi :

Ces gamins, ils n'ont aucune chance de connaitre un jour une vie normale. [Lorsqu'ils sortent de prison,] ils sont à peine descendus du bus qu'ils sont déjà perdus. Ils ne savent pas lire les panneaux dans la rue, la personne qui devait venir les chercher - leur père, un parent - n'arrive pas. Ils ont l'impression de puer la prison. On les traite comme n'importe quel autre détenu de l'aide sociale. [C'est] comme ces gamins qui bavent de l'autre côté de la vitrine du magasin de jouets ${ }^{18}$.

Comme je m'interrogeais sur les contraintes structurelles plus générales qui pesaient sur ces situations, je lui ai demandé s'il pensait que cela avait à voir avec la classe. «Oui, m'a-t-il répondu, Mais c'est presque invisible [pour moi et pour eux]. Ils ont l'habitude qu'on les fasse toujours passer en dernier. La seule façon qu'ils ont trouvée d'attirer l'attention, c'est d'être destructeurs. » Un prisonnier qui n'avait pas vingt ans développait à peu de chose près la même analyse, d'où il ressortait qu'il n'avait aucun espoir en l'avenir :

Je suis assez rebelle et anti-social, assez violent... Je n'aime pas trop les règles... Il y a pas mal de chances que je revienne en prison... Je parle bien, [mais] agir, c'est autre chose. Je sors bientôt [de prison], mais j'ai nulle part où aller... J'ai pas beaucoup de choses pour moi... Je suis prisonnier, et personne ne voudra me donner du travail. C'est terrible à dire, mais je suis drogué, je suis alcoolique. J'aime bien faire ce que je veux. Et c'est vrai que je n'ai pas beaucoup de contrôle sur moi-même. J'ai pas eu la vie facile, et je pourrais mettre ça en avant comme excuse pour rester toute ma 
vie un voyou et un tocard alcoolique. Je voudrais bien changer, mais qu'est-ce que j'ai vraiment à y gagner ? Ceux qui se sont rangés, qui ont un boulot, c'est dur pour eux, ils s'ennuient... Est-ce que c'est la vie que je veux? Il n'y a personne qui m'aime, alors ça servirait à quoi ?

À propos des jeunes prisonniers comme celui-ci, et toujours pour répondre à ma question, le membre du personnel de la prison ajouta :

[Tout ça, c'est une histoire de] plaisir et de douleur... Ces gars-là ne font pas le lien avec les conséquences. On n'arrête pas de les traiter d'idiots, depuis toujours. Ils n'ont jamais rien eu, on pouvait tout leur enlever à n'importe quel moment. [Vous et moi,] on voit bien la relation de cause à effet. On pense à nos petits-enfants à venir. Eux, ils ne pensent pas six mois en avant.

Comme Bentham au XVIII ${ }^{\mathrm{e}}$ siècle, le problème de ce que cet homme considère comme les «classes dangereuses » est leur incapacité à raisonner, non pas parce qu'elles en seraient structurellement incapables, mais parce que leur environnement ne leur a jamais appris pourquoi elles devraient le faire. Le prisonnier « idiot » - comme le jeune homme qui se qualifie lui-même ainsi est le premier à le dire - est capable de manifester ses capacités en théorie, mais il est trop jeune et trop corrompu par son environnement pour le faire en pratique. La longue discussion que j'eus avec ce prisonnier manifestait clairement qu'il était tout à fait conscient des effets de ses actes : ses addictions, son désir irrésistible d'exercer un pouvoir sur les autres et l'absence d'incitation à agir autrement allaient inexorablement aboutir à son retour en prison. Il se considérait ainsi comme irrémédiablement abîmé, ce qui concordait avec la vision que l'agent pénitentiaire avait des jeunes gens comme lui. Plus avant dans notre conversation, il évoqua ses problèmes de sommeil et les expériences de dissociation qui le hantaient lorsqu'il était à l'isolement.

Je me vois glisser vers un endroit où je ne veux pas aller... C'est comme si mon esprit cherchait à aller ailleurs. [Quand j'étais petit,] il m'est arrivé un truc vraiment grave, et quand ça arrivait, j'essayais de faire autre chose, de tout bloquer et de partir ailleurs [c'est ce qui se passe ici aussi].

Dans le parloir de l'unité de haute sécurité où se déroulait cette discussion, les approches développementales des professionnels de santé mentale, qui auraient sans doute relevé cet aveu pour en faire grand cas, pouvaient sembler bien loin. Mais une forme d'intervention sommaire était parfois capable de rompre le mélange de dureté et de fragilité véhiculé par le prisonnier. À propos des actes associés au fait de «faire l'idiot», celui-ci avait parlé de la façon dont les gardiens pouvaient l'aider en "lui cri[ant] dessus ». Aussi bien les gardiens que les détenus estimaient que l'exhortation une sorte de coaching de bon sens - était capable de ramener à la raison le prisonnier qui menaçait de faire l'idiot ${ }^{19}$. Rappeler aux prisonniers ce à quoi sont susceptibles de mener leurs actes - comme de nombreux membres du personnel de la prison m'ont dit le faire avec leurs propres enfants - ne requiert pas d'expertise particulière. Il suffit pour cela d'être prêt à intervenir et à appliquer directement les présupposés concernant le choix rationnel qui sont au fondement du système de traitement des infractions. Sam Delano, le détenu que nous avons cité ci-dessus critiquant son compagnon de cellule, fut finalement libéré de l'unité de haute sécurité et intégré à un programme de transition, avec pour perspective de rejoindre le régime général. À peine une semaine après le début du programme, selon un employé de l'unité de haute sécurité qui était soucieux de sa réussite, il avait «fait une bêtise » : il avait modifié son badge pour pouvoir entrer dans la salle de musculation, ce qui lui avait valu d'être inculpé de «faux et usage de faux ». C'est là un bon exemple d'action "idiote » réalisée 
par un individu rationnel qui ne prend pas en compte les conséquences évidentes de ses actes dans le but de réaliser un objectif à court terme. L'employé de l'unité de haute sécurité était alors allé le voir et l'avait «engueulé comme il fallait». Delano avait «reçu le message et depuis il se comport[ait] très bien ». Pour finir, avec le soutien de l'employé pénitentiaire, on avait recommandé son transfert vers une unité moins restrictive.

Un superviseur d'unité de haute sécurité me donna un autre exemple de cette approche à propos d'un détenu dont la conduite irrémédiablement étrange et auto-destructrice mais néanmoins délibérée, de l'avis du personnel de la prison - avait conduit à son maintien à l'isolement.

On n'en fait pas une affaire personnelle. On dit, eh, c'est à toi de choisir. Je lui dis, écoute, on va chacun faire un pas. Tu crois que c'est ça qu'on veut? Tu crois vraiment qu'on veut que tu te retrouves dans cette [situation] humiliante, avec juste une couverture? Sérieusement, réveille-toi! On ne veut pas de ça pour toi. Il faut que tu coopères avec nous, et on pourra avancer. C'est pas plus compliqué que ça.

Un travailleur de santé mentale rapportait une discussion similaire, qu'il qualifiait de "discussion entre un père et son fils", au cours de laquelle il avait dit à un détenu perturbé, larmoyant, qui faisait constamment des allers-retours entre une unité de santé mentale et une unité de haute sécurité : "C'est idiot. Ce [comportement] ne te conduit nulle part, il faut que ça s'arrête. Tu vas réussir à t'en sortir [à propos de sa longue assignation à une unité de haute sécurité] si tu gardes le moral. »

Ces efforts en faveur des détenus qui ont "fait l'idiot " sont la version carcérale des gestes paternels et enveloppants des professionnels en santé mentale. Être idiot, ce n'est ni être fou ni être mauvais, c'est «être sur le point de ». Par l'exhortation, les employés de la prison tentent de ranimer la raison sous-utilisée du prisonnier avant qu'il ne soit assigné à une catégorie inférieure ou transféré vers une unité de haute sécurité ou de santé mentale. Cet effort de modification du comportement né d'une expérience locale et guidé par le bon sens repose sur l'idée que l'intervention d'experts n'est pas nécessaire, dans la mesure où l'accessibilité du détenu à la raison est une qualité humaine évidente. Et étant donné que l'expression «faire l'idiot » renvoie à la vulnérabilité, et non au caractère, les détenus recourent eux aussi à son pouvoir explicatif. La reconnaissance et le traitement des conduites idiotes constituent ainsi l'arrière-plan - tant pour le personnel que pour les détenus - des systèmes plus théoriques de classification et de diagnostic psychiatrique.

\section{BIBLIOGRAPHIE}

Adorno T., 1994, The Stars down to Earth and Other Essays on the Irrational in Culture, New York, Routledge.

American Psychiatric Association (APA), 2000, Diagnostic and Statistical Manual of Mental Disorders, Fourth Edition - Text Revision, Washington D.C., American Psychiatric Association. 
Austin J., Irwin J., 2001, It's about Time, Belmont, Californie, Wadsworth.

Estroff S.E., 1993, Identity, Disability, and Schizophrenia: The Problem of Chronicity, in Lindenbaum S., Lock M. (Eds.), Knowledge, Power, and Practice: The Anthropology of Medicine and Everyday Life, Berkeley, University of California Press, 247-286

Foucault M., 1994 [1984], Qu'appelle-t-on punir ? (texte n 346), in Foucault M. (dir.), Dits et écrits, 1954-1988, Paris, Gallimard.

Gaines A., 1992, From DSM-I to III-R; Voices of Self, Mastery and Other: A Cultural Constructivist Reading of U.S. Psychiatric Classification, Social Science and Medicine, 35, 1, 3-24.

Harcourt B.E, 2009, Repenser le carcéral à travers le prisme de l'institutionnalisation : Sur les liens entre asiles et prisons aux États-Unis, séminaire Gern Paris mars 2008, Champ pénal/Penal field, [en ligne] http://journals.openedition.org/champpenal/7562.

Horwitz A.V., 2002, Creating Mental Illness, Chicago, University of Chicago Press.

Irwin J., 1970, The Felon, Englewood Cliffs, N.J., Prentice Hall.

Irwin J., 1980, Prisons in Turmoil, Boston, Little, Brown.

Lowe D.M., 1995, The Body in Late Capitalist USA, Durham, Duke University Press.

Luhrmann T.M, 2000, Of Two Minds: The Growing Disorder in American Psychiatry, New York, Alfred A. Knopf.

Rhodes, L.A., 1991, Emptying Beds: The Work of an Emergency Psychiatric Unit, Berkeley, University of California Press.

Rhodes L.A., 2000, Taxonomic Anxieties: Axis I and Axis II in Prison, Medical Anthropology Quarterly, 14, 3, 346-373

Rhodes L.A., 2004, Total Confinement: Madness and Reason in the Maximum Security Prison, Berkeley, University of California Press.

Rhodes L.A., 2010, Risking Therapy, The Howard Journal of Criminal Justice, 49, 5, 451-462.

Rhodes L.A., 2015. Qui décide ici ? Le comportementalisme dans le système carcéral américain, Anthropologie \& Santé, 11, [en ligne] http://journals.openedition.org/anthropologiesante/1860, DOI : $10.4000 /$ anthropologiesante. 1860 .

Roberts J.V., Gebotys R.J., 2001, Prisoners of Isolation: Research on the Effects of Administrative Segregation, Canadian Journal of Criminology, January, 85-97.

Rothman D.J., 1998, Perfecting the Prison: United States 1780-1865, in Morris N., Rothman D.J. (Eds.), The Oxford History of the Prison: The Practice of Punishment in Western Society, New-York, Oxford University Press.

Rotman E., 1998, The Failure of Reform: United States 1865-1965, in Morris N., Rothman D.J. (Eds.), The Oxford History of the Prison: The Practice of Punishment in Western Society, New-York, Oxford University Press.

Tucker G., 1998, Putting DSM-IV in Perspective, American Journal of Psychiatry, 155, 2, 159-161.

Wicker T., 1975, A Time to Die, New York, Quadrangle, New York Times Books.

Wilson M., 1993, DSM-III and the Transformation of American Psychiatry: A History, American Journal of Psychiatry, 150, 3, 399-410.

Young A., 1995, The Harmony of Illusions: Inventing Post-Traumatic Stress Disorder, Princeton, Princeton University Press. 


\section{NOTES}

1. Les unités "Supermax », ou unités de très haute sécurité, se sont multipliées aux États-Unis au cours des années 1980 dans le but officiel de séparer de la détention classique les détenus les plus violents. Ces unités reposent sur le principe de la déprivation sensorielle et du confinement solitaire : les contacts humains entre personnes détenues et avec les personnels de surveillance sont minimisés, les activités et sorties réduites au minimum, la surveillance visuelle constante.

2. À la fin des années 1990, le renouveau des travaux empiriques menés sur la santé mentale a coïncidé avec la diffusion de l'anthropologie psychiatrique américaine récente, sous la forme de traduction ou d'importation, dont faisait partie Emptying beds (Rhodes, 1991). Quelques années plus tard, Total Confinement (Rhodes, 2004) a été lu également par les chercheurs travaillant sur la prison, notamment grâce à l'impulsion de Yasmine Bouagga qui avait participé à la recherche menée dans le Supermax. Lorna Rhodes a depuis été invitée à plusieurs reprises dans des colloques organisés en France pour l'allocution d'ouverture du colloque «Traitements et contraintes : approches empiriques des dispositifs de prise en charge institutionnelle ", 14 et 15 juin 2012, à l'université Paris 13 à Bobigny, organisé par le collectif de jeunes chercheurs Traitements \& Contraintes (http://www.traitements-contraintes.org/) ; ou encore l'intervention de clôture lors du colloque "L'enfermement au prisme des sciences sociales : rapprocher les lieux, confronter les approches", du 16 au 19 octobre 2013 à l'université de Bordeaux-Pessac, organisé par l'ANR TerrFerme (https://terrferme.hypotheses.org/colloque-2013).

3. Cet entretien s'inscrit en quelque sorte dans la continuité d'un article de Bernard E. Harcourt paru il y a presque 10 ans dans la revue Champ pénal (Harcourt, 2009).

4. Cette déstabilisation apparaît dans les textes les plus tardifs de Michel Foucault au sujet de la prison. En 1984, il propose ainsi de porter l'attention non pas seulement sur les finalités de la prison, mais également sur ses effets et ses usages, les deux derniers pouvant venir contredire les premières (Foucault, 1984).

5. Le paradigme biologique consiste à appréhender les troubles mentaux avant tout comme un dysfonctionnement cérébral, plutôt que comme le résultat de processus psychodynamiques. Il s'agit, selon ce paradigme, de faire de la psychiatrie une discipline médicale scientifique, au sens d'une discipline appuyée sur des preuves empiriques (evidence-based medicine).

6. Notons ici que Michel Foucault s'est intéressé, à l'occasion d'une visite de la prison d'Attica (dans l'État de New York) quelques années après les émeutes qui s'y sont produites, au système d'exclusion produit par les prisons de haute sécurité. L'un des enjeux centraux de cette prison se situe selon lui dans le rôle problématique que jouent ceux qui travaillent à la réhabilitation des personnes détenues, et tout particulièrement les psychologues, qui tout à la fois aident les détenus et participent à la légitimation du système. Lorna Rhodes consacre une partie de Total Confinement à analyser, dans ses ramifications les plus subtiles, cette présence ambivalente des psychiatres, psychologues et des savoirs sur lesquels ils s'appuient.

7. Dans cette communication non publiée, intitulée « Repetition, Entropy and the Institutional Interior », Lorna Rhodes propose une réflexion sur le caractère cyclique du changement dans les institutions dites «totales». Elle identifie trois types idéaux, vers lesquelles s'orientent les vagues successives de réformes : la « petite société » (small society), le " panoptique » (panopticon) et le «camp» (camp). Voir le site de la conférence: https://terrferme.hypotheses.org/ colloque-2013.

8. La prison de Grendon (Angleterre), ouverte dans les années 1960, est l'un des établissements pénitentiaires les plus étudiés du Royaume-Uni et fait figure de "prison modèle ». Cette prison dite "thérapeutique » accueille environ 250 détenus répartis en petites communautés d'environ 40 personnes organisées autour d'un emploi du temps réglé comprenant de nombreuses activités collectives («community meetings») visant à promouvoir les «compétences sociales et relationnelles » des personnes détenues. 
9. Objet de controverse en raison de la conception biomédicale de la psychiatrie qu'il véhicule, cette classification fait cependant office d'outil de référence pour la recherche internationale en psychiatrie et la cotation de l'activité clinique des psychiatres aux États-Unis et dans de nombreux pays occidentaux.

10. Si les personnes affectées de graves pathologies mentales doivent recevoir un traitement selon la loi, cette extension extrêmement large du traitement décrite par Irwin serait aujourd'hui considérée comme un luxe inutile. D'autres changements ont également contribué à la restriction de ce qu'on entend par traitement aujourd'hui. L'un est que la psychiatrie elle-même a beaucoup changé depuis l'époque de ces «solutions aux problèmes psychologiques » dans les prisons californiennes : sa perspective est devenue plus médicale et biologique. L'incarcération a également changé: elle s'est professionnalisée et bureaucratisée, avec des systèmes et des procédures de classification centralisés et fondés sur « des méthodes ou un savoir scientifiques ostensiblement valides » (Austin, Irwin, 2001, 98).

11. Le manuel diagnostique a pris une importance croissante du fait de son utilisation comme base pour les demandes d'indemnisation auprès des assurances. Voir (Lowe, 1995), pour une réflexion critique sur la façon dont les catégories psychiatriques et les intervenants en psychiatrie ont pénétré dans de nombreux champs institutionnels.

12. On trouvera une version de ce récit dans mon article: "Taxonomic Anxieties " (Rhodes, 2000).

13. Environ deux semaines après son admission, j'ai de nouveau rencontré Mullen. Il m'a expliqué qu'il avait peur d'être intégré au régime ordinaire et qu'il s'inquiétait de ce que les détenus plus solides ne s'aperçoivent de son anxiété.

14. Dans la période qui a suivi l'ère du traitement décrite par Irwin, laquelle était structurée par des orientations «thérapeutiques" - et souvent développementales, la psychiatrie a connu une révolution classificatoire visible à travers les changements intervenus entre les différentes éditions du DSM. Les premières éditions reposaient sur un modèle médical des troubles mentaux et incluaient des éléments d'étiologie, tandis que les deux dernières éditions (III et IV) sont essentiellement descriptives et s'attachent avant tout au comportement. On trouvera une analyse de l'histoire, de l'usage actuel et des implications théoriques de la classification du DSM dans (Gaines, 1992 Horwitz, 2002 ; Wilson, 1993 ; Young, 1995).

15. Les trois autres axes traitent de condition physique, de facteurs de stress psychosociaux et de l'évaluation du fonctionnement. La classification du DSM est pour l'essentielle indépendante de tout contexte : les "facteurs de stress » et les "vulnérabilités » sont certes mentionnés, mais l'individu est visé uniquement en tant qu'ensemble de caractéristiques. Le résultat est de naturaliser les catégories, de telle sorte qu'elles «prennent l'aspect de quelque chose [...] qui n'aurait pas été fait de main humaine» (Wilson, 1993, 408). Les critiques du DSM au sein de la psychiatrie soulignent que cette approche encourage les praticiens à minimiser l'importance de l'histoire du patient pour «rechercher les symptômes nécessaires à l'établissement d'un diagnostic » (Tucker, 1998, 160). Ou, pour reprendre la formule d'un travailleur de santé mentale en prison : " Le problème du diagnostic, c'est de parvenir à y faire entrer le patient ».

16. Comme le remarquent nombre d'observateurs et de patients, les maladies mentales chroniques comme la schizophrénie finissent en réalité par devenir indémêlables de la personnalité des malades. Pour une réflexion puissante sur ce point, voir (Estroff, 1993).

17. Ce même gardien avait également donné l'exemple d'un détenu qui en avait dénoncé un autre sans réfléchir que « si on est pris à balancer, ça peut faire mal ».

18. On relèvera le lapsus significatif de sa remarque : "n'importe quel autre détenu de l'aide sociale ». La protection destinée aux « démunis » à l'extérieur se mêle à celle de la prison : dans un cas comme dans l'autre, le détenu qui "pue la prison» ne parvient pas à échapper à son assignation à la catégorie de démuni, « abîmé » par la vie. 
19. Ainsi, un prisonnier qui avait passé plusieurs années dans une unité de haute sécurité décrivait comment, réintégré au régime ordinaire, il s'efforçait de convaincre son compagnon de cellule « idiot » d'assumer sa part des tâches ménagères.

\section{AUTEURS}

\section{LORNA RHODES}

Professeure émérite de l'université de Washington

lrhodes[at]uw.edu

\section{CAMILLE LANCELEVÉE}

Docteure en sociologie de l'Institut de recherches interdisciplinaires sur les enjeux sociaux (Iris, EHESS, Paris)

Chargée de recherches à la fédération régionale de recherches en psychiatrie et en santé mentale (F2RSM Psy, Lille)

camille.lancelevee[at]gmail.com

\section{LIVIA VELPRY}

Maîtresse de conférences à l'Université Paris 8-Saint Denis

Chercheure au Cermes3 (Université Paris Descartes, EHESS, CNRS, INSERM)

livia.velpry[at]univ-paris8.fr 